\title{
Erratum to: Reliability and validity of the Taiwan (Mandarin Chinese) version of the chronic respiratory questionnaire
}

\author{
Nai-Hsin Meng • Fei-Na Chen • Sui-Foon Lo • \\ Wei-Erh Cheng
}

Published online: 25 June 2011

(C) Springer Science+Business Media B.V. 2011

\section{Erratum to: Qual Life Res}

DOI 10.1007/s11136-011-9906-7

The third author's name was misspelled in the original publication. The name is correct in this erratum.

The online version of the original article can be found under doi:10.1007/s11136-011-9906-7.

N.-H. Meng · S.-F. Lo

Department of Physical Medicine and Rehabilitation,

China Medical University Hospital, Taichung, Taiwan,

Republic of China

\section{N.-H. Meng}

School of Medicine, China Medical University,

Taichung, Taiwan, Republic of China

\section{F.-N. Chen}

Department of Social Medicine, College of Medicine, China

Medical University, Taichung, Taiwan, Republic of China

W.-E. Cheng $(\bowtie)$

Division of Pulmonary and Critical Care Medicine, Department of Internal Medicine, China Medical University Hospital, No. 2,

Yude Rd, Taichung, Taiwan, Republic of China

e-mail: weierh.cheng@gmail.com

\section{W.-E. Cheng}

Department of Respiratory Therapy, College of Health Care,

China Medical University, Taichung, Taiwan, Republic of China 